\title{
Quaternion Analysis for Generalized Electromagnetic Fields of Dyons in Isotropic Medium
}

\author{
July 4, 2021 \\ (1) Department of Physics \\ Govt. P. G. College \\ Pithoragarh -(UA), INDIA \\ ${ }^{(2)}$ Department of Physics \\ Kumaun University \\ S. S. J. Campus \\ Almora-263601 (UA), INDIA \\ Email:-(1) jgaria@indiatimes.com \\ ${ }^{(2)}$ ps_bisht123@rediffmail.com \\ (2) ops_negi@yahoo.co.in.
}

Jivan Singh ${ }^{(1)}$, P. S. Bisht ${ }^{(2)}$ and O. P. S. Negi( ${ }^{(2)}$

\begin{abstract}
Quaternion analysis of time dependent Maxwell's equations in presence of electric and magnetic charges has been developed and the solutions for the classical problem of moving charges (electric and magnetic) are obtained in unique, simple and consistent manner.
\end{abstract}

\section{Introduction}

It is believed that in spite of the recent potential importance of magnetic monopoles [1], [2], [3] and dyons [4] towards the quark confinement problem [5] of quantum choromodynamics, possible magnetic condensation of vacuum [6], CP-violation [7], their role as catalyst in proton decay [8] and the current grand unified theories [9], the formalism necessary to describe them has been clumsy and manifestly non-covariant. Keeping in view the potential importance of monopoles and the results of Witten [7] that monopoles are necessarily dyons, we [10, 11] have also constructed a self-consistent co-variant theory of generalized electromagnetic fields associated with dyons each carrying the generalized charge as complex quantity with its real and imaginary part as electric and magnetic constituents. On the other hand quaternions were invented by Hamilton [12] to extend the theory of Complex numbers to three dimensions. Maxwell's Equations of electromagnetism were rewritten in terms of quaternions [13]. Finklestein et al [14] developed the quaternionic quantum mechanics and Adler [15] described the theory of the algebraic structure of quantum choromodynamics for strong interactions. Various aspects of quaternions are 
discussed by Morita [16] towards the kinematical structure of Poincare gauge theory and the left-right Weinberg-Salam theory of quantum choromodynamics. We have also studied [17] the quaternionic formulation for generalized field equations of dyons in unique, simpler and compact notations. Quaternion non-Abelian gauge theory has also been consistently discussed [18] to maintain the structural symmetry between the theory of linear gravity and electromagnetism. It is also shown that quaternion formalism characterises the Abelian and non-Abelian gauge structures [19] of dyons in terms of real and imaginary constituents of quaternion basis elements. Altenatively, Kravchenko [20] and his coworkers have consistently analysed the Maxwell's equations for time-dependent electromagnetic fields in homogeneous (isotropic) and chiral medium. Extending this, we 21] have also derived the generalised Maxwell's-Dirac equation in the homogenous (isotropic) medium. It has been shown that the field equations of dyons also remains invariant under the duality transformations in isotropic homogeneous medium and the equation of motion reproduces the rotationally symmetric gauge invariant angular momentum of dyons. In order to extend the theory of monopoles and dyons in isotropic medium and consequently the relevance of quaternion formalism of dyons, in the present paper we have undertaken the study of the quaternion analysis of time dependent Maxwell's equations in presence of electric and magnetic charges and the solution for the classical problem of moving sources are obtained in unique, simpler and consistent manner. Quaternion forms of potential current, field equation and equation of motion are developed in compact manner and it is imphasized that the quantum equations in terms of quaternions are invariant under quaternion, Lorentz and duality transformations. It has also been discussed that the quaternion analyticity of dyons in isotropic medium reproduces the results of Kravchenko [20] in the absence of magnetic monopole and accordingly this theory can be described symmetrically for pure monopole in the absence of electric charge or vice versa.

\section{Generalized field equation of dyons in Homogeneous Medium}

Assuming the existence of magnetic monopoles, we may write the following form [22] of symmetric generalized Maxwell-Dirac differential equations [1, 21] in free space in SI units $(c=\hbar=1)$ as;

$$
\begin{aligned}
\vec{\nabla} \cdot \vec{D} & =\rho_{e} \\
\vec{\nabla} \cdot \vec{B} & =\mu_{0} \rho_{m} \\
\vec{\nabla} \times \vec{E} & =-\frac{\partial \vec{B}}{\partial t}-\frac{\overrightarrow{j_{m}}}{\epsilon_{0}} \\
\vec{\nabla} \times \vec{H} & =\frac{\partial \vec{D}}{\partial t}+\overrightarrow{j_{e}}
\end{aligned}
$$

where $\rho_{e}$ and $\rho_{m}$ are respectively the electric and magnetic charge densities while $\overrightarrow{j_{e}}$ and $\overrightarrow{j_{m}}$ are the corresponding current densities, $\vec{D}$ is electric induction vector, $\vec{E}$ is electric field, $\vec{B}$ is magnetic induction vector and $\vec{H}$ is magnetic field. Here we asume the homogenous (isotropic) medium with the following definitions [15],

$$
\vec{D}=\epsilon \vec{E} \quad\left(\epsilon=\epsilon_{0} \epsilon_{r}\right)
$$

and 


$$
\vec{B}=\mu \vec{H}\left(\mu=\mu_{0} \mu_{r}\right)
$$

where $\epsilon_{o}$ the free space permitivity, $\mu_{0}$ is the permeability of free space and $\epsilon_{r}$ and $\mu_{r}$ are defined respectively as relative permitivity and permeability in electric and magnetic fields. On using equations (2) and (3), equation (112314) takes the following differential form [19],

$$
\begin{aligned}
\vec{\nabla} \cdot \vec{E} & =\frac{\rho_{e}}{\epsilon} \\
\vec{\nabla} \cdot \vec{B} & =\mu \rho_{m} \\
\vec{\nabla} \times \vec{E} & =-\frac{\partial \vec{B}}{\partial t}-\frac{\overrightarrow{j_{m}}}{\epsilon} \\
\vec{\nabla} \times \vec{B} & =\frac{1}{v^{2}} \frac{\partial \vec{E}}{\partial t}+\mu \overrightarrow{j_{e}}
\end{aligned}
$$

Differential equations (12/3]4) are the generalised field equations of dyons in homogenous medium and the electric and magnetic fields are corresponding called generalised electromegnetic fields of dyons. These electric and magnetic fields of dyons are expresed in following differential form in homogenous medium in terms of two potentials [11] as,

$$
\begin{aligned}
\vec{E} & =-\vec{\nabla} \phi_{e}-\frac{\partial \vec{C}}{\partial t}-\vec{\nabla} \times \vec{D} \\
\vec{B} & =-\vec{\nabla} \phi_{m}-\frac{1}{v^{2}} \frac{\partial \vec{D}}{\partial t}+\vec{\nabla} \times \vec{C}
\end{aligned}
$$

where $\left\{C^{\mu}\right\}=\left\{\phi_{e}, v \vec{C}\right\}$ and $\left\{D^{\mu}\right\}=\left\{v \phi_{m}, \vec{D}\right\}$ are the two four-potentials associated with electric and magnetic charges.

Let us define the complex vector field $\vec{\psi}$ in the following form

$$
\vec{\psi}=\vec{E}-i v \vec{B}
$$

Equations (516) and (7) lead to the following relation between generalized field and the components of generalized four-potential as,

$$
\vec{\psi}=-\frac{\partial \vec{V}}{\partial t}-\vec{\nabla} \phi-i v(\vec{\nabla} \times \vec{V})
$$

where $\left\{V_{\mu}\right\}$ is the generalised four-potential of dyons in homogenous medium and defined as

$$
V_{\mu}=\{\phi, \vec{V}\}
$$

i.e.

$$
\phi=\phi_{e}-i v \phi_{m}
$$

and 


$$
\vec{V}=\vec{C}-i \frac{\vec{D}}{v}
$$

Maxwell's field equation (78]9]10 may then be written in terms of generalized field $\vec{\psi}$ as

$$
\begin{aligned}
\vec{\nabla} \cdot \vec{\psi} & =\frac{\rho}{\epsilon} \\
\vec{\nabla} \times \vec{\psi} & =-i v\left(\mu \vec{j}+\frac{1}{v^{2}} \frac{\partial \vec{\psi}}{\partial t}\right)
\end{aligned}
$$

where $\rho$ and $\vec{j}$ are the generalized charge and current source densities of dyons in homogenous medium given by

$$
\begin{aligned}
\rho & =\rho_{e}-i \frac{\rho_{m}}{v} \\
\vec{j} & =\overrightarrow{j_{e}}-i v \overrightarrow{j_{m}} .
\end{aligned}
$$

Using equation (12) we introduce a new parameter $\vec{S}$ (i.e. the field current) as

$$
\vec{S}=\square \vec{\psi}=-\mu \frac{\partial \vec{j}}{\partial t}-\frac{1}{\epsilon} \vec{\nabla} \rho-i v \mu(\vec{\nabla} \times \vec{j})
$$

where $\square$ is the D'Alembertian operator and expressed as

$$
\square=\frac{1}{v^{2}} \frac{\partial^{2}}{\partial t^{2}}-\nabla^{2}=\frac{1}{v^{2}} \frac{\partial^{2}}{\partial t^{2}}-\frac{\partial^{2}}{\partial x^{2}}-\frac{\partial^{2}}{\partial y^{2}}-\frac{\partial^{2}}{\partial z^{2}} .
$$

where $v$ is the speed of electromagnetic wave in homogenous isotropic medium. In terms of complex potential the field equation is written as

$$
\begin{aligned}
\square \phi & =v \mu \rho \\
\square \vec{V} & =\mu \vec{j} .
\end{aligned}
$$

We write the following tensorial form of generalized Maxwell's -Dirac equations of dyons in homogenous medium as

$$
\begin{aligned}
& F_{\mu \nu, \nu}=j_{\mu}^{e} \\
& F_{\mu \nu, \nu}^{d}=j_{\mu}^{m} .
\end{aligned}
$$

Defining generalized field tensor of dyon as

$$
G_{\mu \nu}=F_{\mu \nu}-i v F_{\mu \nu}^{d}
$$

One can directly obtain the following generalized field equation of dyon in homogenous (isotropic) medium i.e. 


$$
\begin{aligned}
& G_{\mu \nu, \nu}=j_{\mu} \\
& G_{\mu \nu, \nu}^{d}=0 .
\end{aligned}
$$

The Lorentz four-force equation of motion for dyons in homogenous (isotropic) medium as

$$
f_{\mu}=m_{0} \ddot{x_{\mu}}=\operatorname{Re} Q *\left(G_{\mu \nu} u^{\nu}\right)
$$

where $R e$ donetes real part, $\ddot{x_{\mu}}$ is the four-acceleration and $\left\{u^{\nu}\right\}$ is the four-velocity of the particle and $Q$ is the generalized charge of dyon in isotropic medium.

\section{Quaternion analysis for generalised Maxwell's equation in homogenous medium}

A quaternion is defined as

$$
q=q_{0} e_{0}+q_{1} e_{1}+q_{2} e_{2}+q_{3} e_{3}
$$

where $q_{0}, q_{1}, q_{2}, q_{3}$ are real numbers and called the components of the quaternion $q$ and the quaternion units $e_{0}, e_{1}, e_{2}, e_{3}$ satisfy the following multiplication rules;

$$
\begin{aligned}
e_{0}^{2} & =1 \\
e_{j} e_{k} & =-\delta_{j k}+\epsilon_{j k l} e_{l}
\end{aligned}
$$

where $\delta_{j k}$ and $\epsilon_{j k l}\left(\mathrm{j}, \mathrm{k}, \mathrm{l}=1,2,3\right.$ and $\left.e_{0}=1\right)$ are respectively the Kronecker delta and three-index Levi-Civita symbol. The sum of two quaternions $\mathrm{p}$ and $\mathrm{q}$ is defined by adding the corresponding components,

$$
p+q=\left(p_{0}+q_{0}\right) e_{0}+\left(p_{1}+q_{1}\right) e_{1}+\left(p_{2}+q_{2}\right) e_{2}+\left(p_{3}+q_{3}\right) e_{3}
$$

and the multiplication of two quaternions is defined as ;

$$
p \cdot q=\left(p_{0} q_{0}-\vec{p} \cdot \vec{q}\right) e_{0}+\sum_{j}\left(p_{j} q_{0}+p_{0} q_{j}+\epsilon_{j k l} p_{k} q_{l}\right) e_{j}
$$

Thus each quaternion $q$ is the sum of a scalar $q_{0}$ and a vector $\vec{q}$,

$$
q=\operatorname{scalar}(q)+\operatorname{vector}(q)=q_{0}+\vec{q}
$$

where $\vec{q}=q_{1} e_{1}+q_{2} e_{2}+q_{3} e_{3}$. Thus the product of two quaternions $p$ and $q$ is also written as

$$
p q=p_{0} q_{0}-\vec{p} \cdot \vec{q}+p_{0} \vec{q}+q_{0} \vec{p}+\vec{p} \times \vec{q}
$$


where the dot and cross indicate, respectively, the usual three dimensional scalar and vector products. For any quaternion, there exists a quaternion conjugate

$$
\bar{q}=q-q_{1} e_{1}-q_{2} e_{2}-q_{3} e_{3}=q_{0}-\vec{q} .
$$

Quaternion conjugate is an automorphism of ring of quaternion i.e.

$$
(\overline{p q})=(\bar{q})(\bar{p}) .
$$

The norm of a quaternion is given as

$$
q \cdot \bar{q}=\bar{q} \cdot q=q_{0}^{2}+q_{1}^{2}+q_{2}^{2}+q_{3}^{2}=|q|^{2} .
$$

The inverse of a quaternion $q$ is also a quaternion

$$
q^{-1}=\frac{\bar{q}}{|q|^{2}} .
$$

Let us define the quaternionic form of differential operator as [19],

$$
\square=\left(-\frac{i}{v} \partial_{t}+D\right)
$$

and

$$
\bar{\square}=\left(-\frac{i}{v} \partial_{t}-D\right)
$$

where $D=e_{1} \partial_{1}+e_{2} \partial_{2}+e_{3} \partial_{3}$. As such we can express the quantum equation associated with generalized four-potential, four current, electric field and magnetic field in terms of quaternionic analysis as

$$
\begin{aligned}
V & =-i \frac{\phi}{v} e_{0}+V_{1} e_{1}+V_{2} e_{2}+V_{3} e_{3} \\
J & =-i \rho v e_{0}+J_{1} e_{1}+J_{2} e_{2}+J_{3} e_{3} \\
E & =E_{1} e_{1}+E_{2} e_{2}+E_{3} e_{3} \\
B & =B_{1} e_{1}+B_{2} e_{2}+B_{3} e_{3} .
\end{aligned}
$$

Operating equation (37) to equation (77) and using equation (27), we get

$$
\bar{\nabla} \psi=i v \mu J=i \sqrt{\frac{\mu}{\epsilon}} J .
$$

Similarly we may operate equation (36) to equation (39) and on using equation (27) we get

$$
\bullet J=S .
$$


where we have used the following subsidiary condition

$$
\vec{\nabla} \cdot \vec{V}+\frac{1}{v^{2}} \frac{\partial \phi}{\partial t}=0
$$

and

$$
\vec{\nabla} \cdot \vec{J}+\frac{\partial \rho}{\partial t}=0
$$

Equation (44) is known as Lorentz gauge condition while equation (45) is referred as continuity equation. $\vec{\psi}$ and $\vec{S}$ are defined as quaternion valued vector functions in the following manner

$$
\begin{aligned}
\psi & =-\psi_{t}-\frac{i}{v}\left(e_{1} \psi_{1}+e_{2} \psi_{2}+e_{3} \psi_{3}\right. \\
S & =-S_{t}-i \sqrt{\frac{\epsilon}{\mu}}\left(e_{1} S_{1}+e_{2} S_{2}+e_{3} S_{3}\right) .
\end{aligned}
$$

Similarly, we may obtain the quaternion conjugate field equations for dyons in homogenous (isotropic) medium as

$$
\begin{aligned}
& \overline{\ominus V}=\bar{\psi} \\
& \overline{\ominus J}=\bar{S}
\end{aligned}
$$

where $\bar{V}, \bar{J}, \bar{\psi}$ and $\bar{S}$ are the quaternion conjugates defined as

$$
\begin{aligned}
\bar{V} & =-i \frac{\phi}{v} e_{0}-\left(V_{1} e_{1}+V_{2} e_{2}+V_{3} e_{3}\right) \\
\bar{J} & =-i \rho v e_{0}-\left(J_{1} e_{1}+J_{2} e_{2}+J_{3} e_{3}\right) \\
\bar{\psi} & =-\psi_{t}+\frac{i}{v}\left(e_{1} \psi_{1}+e_{2} \psi_{2}+e_{3} \psi_{3}\right) \\
\bar{S} & =-S_{t}+i \sqrt{\frac{\epsilon}{\mu}}\left(e_{1} S_{1}+e_{2} S_{2}+e_{3} S_{3}\right) .
\end{aligned}
$$

Hence the quaternion forms of equation (18|19) for generalized potential, equation (23|24) for generalized Maxwell's Dirac equation and Lorentz force equation (25) for dyons in homogeneous medium may be expressed in terms of the following set of quaternion equations in simple, compact and consistent manner.

$$
\begin{aligned}
& \square \bar{\nabla} V=J \\
& {[\boxminus, G]=J} \\
& {[\square, J]=0} \\
& {[u, G]=f}
\end{aligned}
$$

where 


$$
\begin{aligned}
u & =-i u_{0} e_{0}+u_{1} e_{1}+u_{2} e_{2}+u_{3} e_{3} \\
G & =-i G_{0} e_{0}+G_{1} e_{1}+G_{2} e_{2}+G_{3} e_{3} .
\end{aligned}
$$

In equation (57), $u, G$ and $f$ are the quaternionic forms of velocity, generalized field tensor and Lorentz force associated with dyons in homogeneous medium. The elements of $G$ in equation (59) are the following quaternion forms,

$$
\begin{aligned}
G_{0} & =G_{01} e_{1}+G_{02} e_{2}+G_{03} e_{3}, \\
G_{1} & =i G_{10} e_{1}+G_{12} e_{2}+G_{13} e_{3}, \\
G_{2} & =i G_{20} e_{1}+G_{21} e_{2}+G_{23} e_{3}, \\
G_{3} & =i G_{30} e_{1}+G_{31} e_{2}+G_{32} e_{2} .
\end{aligned}
$$

Equations (55, 56 ) and (57) may also be described as

$$
\left[\odot, G_{\mu}\right]=J_{\mu}
$$

and

$$
\left[u, G_{\mu}\right]=f_{\mu}
$$

where $J_{\mu}$ and $f_{\mu}$ are the four current and four force associated with generalized field of dyons in homogeneous (isotropic) medium. Let us factorize the wave operator as the combination of quaternion and its conjugate in the following manner [20],

$$
\left(-\triangle-\frac{1}{v^{2}} \partial_{t}^{2}\right)=\left(-\frac{i}{v} \partial_{t}+D\right)\left(-\frac{i}{v} \partial_{t}-D\right)
$$

Moreover, each solution of the wave equation

$$
\left(\triangle+\frac{1}{v^{2}} \partial_{t}^{2}\right) b=0
$$

can be written in a simple and compact form as the sum of two functions $A$ and $B$, which reduces to following set of differential equations

$$
\begin{aligned}
\left(D-\frac{i}{v} \partial_{t}\right) A & =j_{e} \\
\left(-D-\frac{i}{v} \partial_{t}\right) B & =j_{m}
\end{aligned}
$$

respectively for electric and magnetic charges of a particle described as dyons.

\section{Moving dyons}

Let us define the generalized charge of dyons moving in generalized electromagnetic field as ; 


$$
Q=e-i \frac{g}{v}
$$

where $e$ is electronic charge and $g$ is magnetic charge. Let us assume that a dyon is moving with a velocity $\vec{V}(t)$. Thus, the electric charge density $\rho_{e}$ of a dyon may be expressed as,

$$
\rho_{e}(t, x)=e \delta(x-S(t))
$$

where $S(t)$ is the trajectory of the electron and the current density $\overrightarrow{j_{e}}$ is written as,

$$
\overrightarrow{j_{e}}(t, x)=\vec{V}(t) \rho_{e}(t, x)
$$

We now use equation (66) along with the known solution of the equation i.e.

$$
\left(\triangle+\frac{1}{v^{2}} \partial_{t}^{2}\right) b(t, x)=A(t) \delta(x-S(t))
$$

which may be expressed in terms of the following formula,

$$
b(t, x)=\frac{A(\tau(t))}{4 \pi|x-S(\tau(t))|(1-M(S(t))}
$$

where

$$
M(\tau)=\frac{<\vec{V}(\tau), x-S(\tau)>}{v|x-S(\tau)|}
$$

is called the Mach number and $\tau$ satisfies the equation

$$
\frac{|x-S(\tau)|}{v}-(t-\tau)=0 .
$$

Similarly as the case of electron, let us describe that a monopole constituent of a dyon is also moving with a velocity $\vec{V}(t)$. Then the magnetic charge density $\rho_{m}$ and magnetic current density $\overrightarrow{j_{m}}$ in view of duality transformations $[21,22]$ lead to the following expressions i.e.

$$
\rho_{m}(t, x)=g \delta(x-S(t))
$$

and

$$
\overrightarrow{j_{m}}(t, x)=\vec{V}(t) \frac{\rho_{m}(t, x)}{v^{2}}
$$

Taking into account the explicit form of $\rho_{e}, j_{e}$ and $\rho_{m}, j_{m}$ equation (42) is described as follows:

$$
\bar{\nabla} \psi=\left[\frac{1}{\epsilon}+i v \mu \vec{V}(t)\right] e \delta(x-S(t))-\frac{i}{\epsilon}\left[\frac{1}{v}+i \frac{\vec{V}(t)}{v^{2}}\right] g \delta(x-S(t))
$$


Thus, the purely vectorial biquaternion

$$
\psi(t, x)=\left(-\frac{i}{v} \partial_{t}+D\right) b(t, x)
$$

is a solution of equation (79) if $b$ is a solution of equation (73) with

$$
A(t)=\left[\left(\frac{e}{\epsilon}+i e v \mu \vec{V}(t)\right)-\frac{i}{\epsilon}\left(\frac{g}{v}+i g \frac{\vec{V}(t)}{v^{2}}\right)\right] .
$$

Here $b$ and $A$ both are complex quaternionic functions. Using equation (73/74/75) we obtain

$$
b_{0}(t, x)=\frac{\frac{1}{\epsilon}\left(e-i \frac{g}{v}\right)}{4 \pi|x-S(\tau(t))|(1-M(\tau(t))}=\frac{Q}{4 \pi \epsilon|x-S(\tau(t))|(1-M(\tau(t))}
$$

and

$$
\vec{b}(t, x)=\frac{i\left(e v \mu \vec{V}(t)-i \frac{g}{\epsilon} \frac{\vec{V}(t)}{v^{2}}\right)}{4 \pi|x-S(\tau(t))|(1-M(\tau(t))}=\frac{i v \mu \vec{V}(t) Q}{4 \pi|x-S(\tau(t))|(1-M(\tau(t))} .
$$

Thus, solution (79) reduces to a simple differentiation, i.e.

$$
\psi(t, x)=\left(-\frac{i}{v} \partial_{t}+D\right)\left[\frac{\frac{1}{\epsilon} Q+i v \mu \vec{V}(t) Q}{4 \pi|x-S(\tau(t))|(1-M(\tau(t))}\right] .
$$

We may now introduce the auxiliary functions as

$$
\begin{aligned}
\varphi & =\frac{1}{|x-S(\tau(t))|(1-M(\tau(t))} \\
\zeta & =\frac{1}{4 \pi}\left[\frac{1}{\epsilon} Q+i v \mu \vec{V}(t) Q\right] .
\end{aligned}
$$

Then

$$
\psi=\left(-\frac{i}{v} \partial_{t}+D\right)[\zeta] \cdot \varphi+\left(-\frac{i}{v} \partial_{t}+D\right)[\varphi] \cdot \zeta .
$$

It is easy to see that the scalar part of the expression on the right hand side is zero. Rewriting Maxwell's equations (4) in vector form as follows:

$$
\vec{\psi}=-\frac{i}{v}\left(\partial_{t} \vec{\zeta} \cdot \varphi+\partial_{t} \varphi \cdot \vec{\zeta}\right)+\varphi \vec{\nabla} \times \vec{\zeta}+\zeta_{0} \vec{\nabla} \varphi+[\vec{\nabla} \varphi \times \vec{\zeta}]
$$

By the definition of $\vec{\psi}$ from equation (7), we have,

$$
\vec{E}=-\frac{i}{v}\left(\partial_{t} \vec{\zeta} \cdot \varphi+\partial_{t} \varphi \cdot \vec{\zeta}\right)+\frac{1}{4 \pi \epsilon} Q[\vec{\nabla} \varphi]
$$

and 


$$
v \vec{B}=\varphi \vec{\nabla} \times \vec{\zeta}+[\vec{\nabla} \varphi \times \vec{\zeta}]
$$

To obtain the following generalized electric and magnetic field vectors $\vec{E}$ and $\vec{B}$ in explicit form we have used the following intermidate equalities i.e.

$$
\begin{gathered}
\partial_{t} \vec{\zeta}=i \frac{\left(e v \mu \overrightarrow{V^{\prime}}(\tau)-i \frac{g}{\epsilon} \overrightarrow{V^{\prime}}(\tau)\right)}{4 \pi(1-M(\tau))}, \\
\vec{\nabla} \times \vec{\zeta}=\frac{i}{4 \pi}\left[e v \mu\left(\vec{\nabla} \tau \times \overrightarrow{V^{\prime}}(\tau)\right)-i \frac{g}{\epsilon}\left(\vec{\nabla} \tau \times \overrightarrow{V^{\prime}}(\tau)\right)\right], \\
{[\vec{\nabla} \varphi \times \vec{\zeta}]=\left(v^{2}+\frac{i}{4 \pi}\left\{\frac{e v \mu[(x-s) \times \vec{V}]-i \frac{g}{\epsilon}[(x-s) \times \vec{V}]}{v^{2}|x-s|^{3}(1-M)^{3}}\right\} \times\right.} \\
\left.<v^{2}+<\vec{V}^{\prime}, x-s>-|\vec{V}|^{2}\right) .
\end{gathered}
$$

As such, the solutions of the above problem for a moving dyon in terms of electric and magnetic field vectors of may now be obtained in the following forms in terms of intermidate equalities i.e.,

$$
\begin{aligned}
& \vec{E}=\frac{i}{4 \pi} e \mu\left\{\frac{\overrightarrow{V^{\prime}}(\tau)}{|x-s|(1-M)^{2}}+\left[\frac{(\vec{V}(\tau)|x-s|-\vec{v}|x-s|)}{v|x-s|^{3}(1-M)^{3}}\right]\left(v^{2}+<\overrightarrow{V^{\prime}}, x-s>-|\vec{V}|^{2}\right)\right\} \\
& +\frac{g}{4 \pi \epsilon v}\left\{\frac{\overrightarrow{V^{\prime}}(\tau)}{|x-s|(1-M)^{2}}+\left[\frac{\left(\overrightarrow{V^{\prime}}(\tau)|x-s|-\vec{v}|x-s|\right)}{v|x-s|^{3}(1-M)^{3}}\right]\left(v^{2}+<\overrightarrow{V^{\prime}}, x-s>-|\vec{V}|^{2}\right)\right\}
\end{aligned}
$$

and

$$
\begin{aligned}
\vec{B} & =\frac{e \mu}{4 \pi}\left\{\frac{\left[(x-s) \times \overrightarrow{V^{\prime}}(\tau)\right]}{v|x-s|^{2}(1-M)^{2}}+\frac{[(x-s) \times \vec{v}(\tau)]}{v^{2}|x-s|^{3}(1-M)^{3}}\left(v^{2}+<\overrightarrow{V^{\prime}}, x-s>-|\vec{V}|^{2}\right)\right\} \\
& -\frac{i g}{v \epsilon}\left\{\frac{\left[(x-s) \times \overrightarrow{V^{\prime}}(\tau)\right]}{v|x-s|^{2}(1-M)^{2}}+\frac{[(x-s) \times \vec{v}(\tau)]}{v^{2}|x-s|^{3}(1-M)^{3}}\left(v^{2}+<\overrightarrow{V^{\prime}}, x-s>-|\vec{V}|^{2}\right)\right\} .
\end{aligned}
$$

These expressions, associated with the solutions of generalized field equations of dyons, reduce to the solutions of usual elecrtic and magnetic field vectors in the absence of magnetic (electric) charge on dyons similar to those described by Kravchenko [20] or vice versa.

\section{References}

[1] Dirac P. A .M, PRC. R. Soc. London, $\underline{\mathbf{A 1 3 3}}$ (1931), 60.

[2] t' Hooft G., Nucl.Phys., B138 (1978),1.

[3] Polykov A. M., JEPT Lett, $\underline{\mathbf{2 0}}$ (1974), 194. 
[4] Julia B. and Zee A., Phys. Rev., D11 (1975), 2227.

[5] Cho Y. M., Phys., Rev., D21 (1980), 1080.

[6] t'Hooft G., Nucl.Phys., B190 (1981),455.

[7] Witten E., Phy. Lett., $\underline{B 86}$ (1979), 283.

[8] Rubakov V.A., Nucl. Phy., B203 (1982), 211.

[9] Dokos C. and Tomaros T., Phy. Rev., D21 (1980), 2940.

[10] Bisht P. S ., Negi O. P.S. and Rajput B. S., Ind. J. of Pure and Appl. Phys., $\underline{\mathbf{2 8}}$ (1990), 157 ; $\underline{\mathbf{2 4}}$ (1993), 543 .

[11] Bisht Shalini, Bisht P. S. and Negi O. P. S., Nuovo Cimento, B113, (1998) 1449.

[12] Hamilton W.R. Elements of quaternions, Vol. I, II and III (Chelsea, New York), 1899.

[13] Rajput B. S., Kumar S. R. and Negi O. P. S., Lett Nuovo Cimento, $\underline{\mathbf{3 6}}$ (1983), 75.

[14] Finkelstein D., Jauch J. M., Schiminovish S. and Speises D., J. Math. Phys., $\underline{30}$ (1962), 267.

[15] Adler S. L., Commun. Math. Phys., 104 (1986), 611.

[16] Morita K., Prog. Theory Phys., $7 \underline{73}$ (1985), 999.

[17] Bisht P.S., Negi O.P.S and Rajput B. S., Ind..J Pure and Appl. Phys., $\underline{\mathbf{2 9}}$ (1991), 457.

[18] Bisht P.S., Negi O.P.S and Rajput B. S., Prog. Theor. Phys., $\underline{\mathbf{8 5}}$ (1991), 151.

[19] Bisht P.S., Negi, O.P.S and Rajput B.S., Int. J. Theor. Phys. 32 (1993), 2099.

[20] Kravchenko, V.V., "Applied Quaternionic Analysis, Research and Exposition in Mathematics", Heldermann Press, Germany, 28 (2003) and reference therein; "Quaternionic equation for electromagnetic fields in inhomogeneous media", math-ph/0202010; Sergei M. Grudsky, Kira V. Khmelnytskaya,Vladislav V. Kravchenko, "On a quaternionic Maxwell equation for the time-dependent electromagnetic field in a chiral media", math-ph/0309062.

[21] Singh Jivan, Bisht P. S. and Negi O. P. S. , "Generalized Electromagnetic Fields of Dyons in Isotropic Medium", arXiv:hep-th/0611208.

[22] Barker W. A. and Graziani Frank, Am. J. Phys., $\underline{46}$ (1978),1111. 